\title{
Probing technicolor theories with staggered fermions
}

\section{Zoltán Fodor}

Department of Physics, University of Wuppertal, Gauss Strasse 20, D-42119, Germany

E-mail: fodor@bodri.elte.hu

\section{Kieran Holland*}

Department of Physics, University of the Pacific

3601 Pacific Ave, Stockton CA 95211, USA

E-mail: khollandepacific.edu

\section{Julius Kuti}

Department of Physics 0319, University of California, San Diego 9500 Gilman Drive, La Jolla CA 92093, USA

E-mail: jkuti@ucsd.edu

\section{Dániel Nógrádi}

Department of Physics 0319, University of California, San Diego

9500 Gilman Drive, La Jolla CA 92093, USA

E-mail: nogradielorentz.leidenuniv.nl

\section{Chris Schroeder}

Department of Physics 0319, University of California, San Diego

9500 Gilman Drive, La Jolla CA 92093, USA

E-mail: crsephysics.ucsd.edu

One exciting possibility of new physics beyond the Standard Model is that the fundamental Higgs sector is replaced by a strongly-interacting gauge theory, known as technicolor. A viable theory must break chiral symmetry dynamically, like in QCD, to generate Goldstone bosons which become the longitudinal components of the $W^{ \pm}$and $Z$. By measuring the eigenvalues of the Dirac operator, one can determine if chiral symmetry is in fact spontaneously broken. We simulate $S U$ (3) gauge theory with $n_{s}=2$ and 3 staggered flavors in the fundamental representation, corresponding to $N_{f}=8$ and 12 flavors in the continuum limit. Although our first findings show that both theories are consistent with dynamically broken chiral symmetry and QCD-like behavior, flavor breaking effects in the spectrum may require further clarifications before final conclusions can be drawn. We also compare various improved staggered actions, to suppress this potentially large flavor breaking.

The XXVI International Symposium on Lattice Field Theory

July 14 - 19, 2008

Williamsburg, Virginia, USA

\footnotetext{
*Speaker.
} 


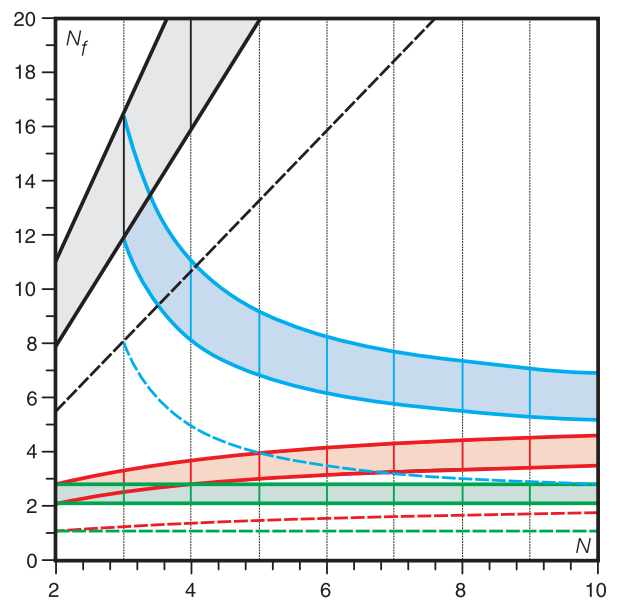

Figure 1: The conformal window for $S U(N)$ gauge theories with $N_{f}$ techniquarks in various representations, from [3]. The shaded regions are the windows, for fundamental (gray), 2-index antisymmetric (blue), 2-index symmetric (red) and adjoint (green) representations.

\section{Introduction}

The LHC will probe the mechanism of electroweak symmetry breaking. A very attractive alternative to the standard Higgs mechanism, with fundamental scalars, involves new stronglyinteracting gauge theories, known as technicolor [1], 2]. Such models avoid difficulties of theories with scalars, such as triviality and fine-tuning. Chiral symmetry must be spontaneously broken in a technicolor theory, to provide the technipions which generate the $W^{ \pm}$and $Z$ masses and break electroweak symmetry. Although this duplication of QCD is appealing, precise electroweak measurements have made it difficult to find a viable candidate theory. It is also necessary to enlarge the theory (extended technicolor) to generate quark masses, without generating large flavor-changing neutral currents, which is challenging.

Technicolor theories have lately enjoyed a resurgence, due to the exploration of various techniquark representations [3]. Feasible candidates have fewer new flavors, reducing tension with electroweak constraints. If a theory is almost conformal, it is possible this generates additional energy scales, which could help in building the extended technicolor sector. There are estimates of which theories are conformal for various representations, shown in Fig. 1. For $S U(N)$ gauge theory, if the number of techniquark flavors is less than some critical number, conformal and chiral symmetries are broken and the theory is QCD-like. For future model-building, it is crucial to go beyond these estimates and determine precisely where the conformal windows are. There have been a number of recent lattice simulations of technicolor theories, attempting to locate the conformal windows for various representations [4, 5, 6, , , , 8].

\section{Dirac eigenvalues and chiral symmetry}

The connection between the eigenvalues $\lambda$ of the Dirac operator and chiral symmetry breaking 


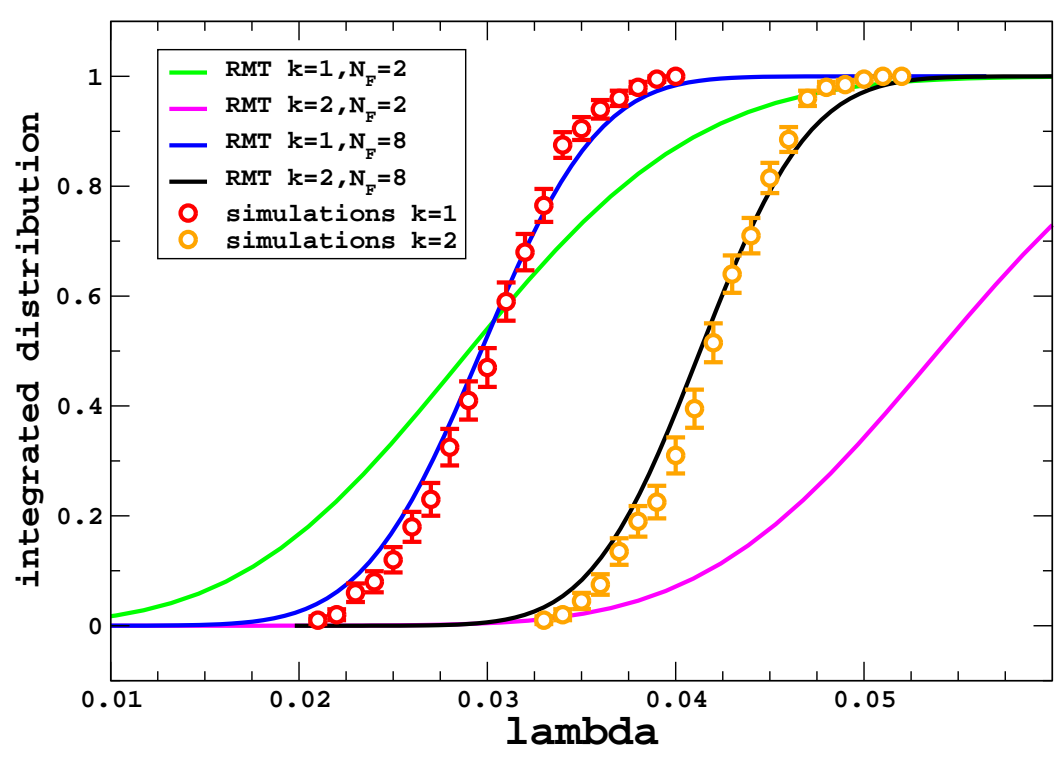

Figure 2: The integrated distribution of the two lowest eigenvalue quartets, from simulations of $n_{s}=2$ Asqtad staggered flavors. This is compared to RMT with $N_{f}=2$ and 8, corresponding to the strong and weak coupling limits.

is succinctly given in the Banks-Casher relation [9],

$$
\Sigma=-\langle\bar{\Psi} \Psi\rangle=\lim _{\lambda \rightarrow 0} \lim _{m \rightarrow 0} \lim _{V \rightarrow \infty} \frac{\pi \rho(\lambda)}{V} .
$$

To generate a non-zero density $\rho(0)$, the smallest eigenvalues must become densely packed as the volume increases, with an eigenvalue spacing $\Delta \lambda \approx 1 / \rho(0)=\pi /(\Sigma V)$. This allows a crude estimate of the quark condensate $\Sigma$. One can do much better by exploring the $\varepsilon$-regime: If chiral symmetry is spontaneously broken, tune the volume and quark mass such that

$$
\frac{1}{F_{\pi}} \ll L \ll \frac{1}{m_{\pi}}
$$

so that the pion is much lighter than the physical value, and finite-volume effects are dominant [10]. The chiral Lagrangian,

$$
\mathscr{L}=\frac{F_{\pi}^{2}}{4} \operatorname{Tr}\left(\partial_{\mu} U \partial_{\mu} U^{\dagger}\right)+\frac{\Sigma}{2} \operatorname{Tr}\left[M\left(U+U^{\dagger}\right)\right], \quad U=\exp \left[\frac{i \pi^{a} T^{a}}{F_{\pi}}\right]
$$

is dominated by the zero-momentum mode from the mass term and all kinetic terms are suppressed. In this limit, the distributions of the lowest eigenvalues are identical to those of random matrix theory (RMT), a theory of large matrices obeying certain symmetries [11]. To connect with RMT, the eigenvalues and quark mass are rescaled as $z=\lambda \Sigma V$ and $\mu=m \Sigma V$, and the eigenvalue distributions also depend on the topological charge $v$ and the number of quark flavors $N_{f}$. RMT is a very useful tool to calculate analytically all of the eigenvalue distributions. The eigenvalue distributions in various topological sectors are measured via lattice simulations, and via comparison with RMT, the value of the condensate $\Sigma$ can be extracted. This method has been successfully used in a number of lattice QCD studies, for example in dynamical overlap fermion simulations [12]. 


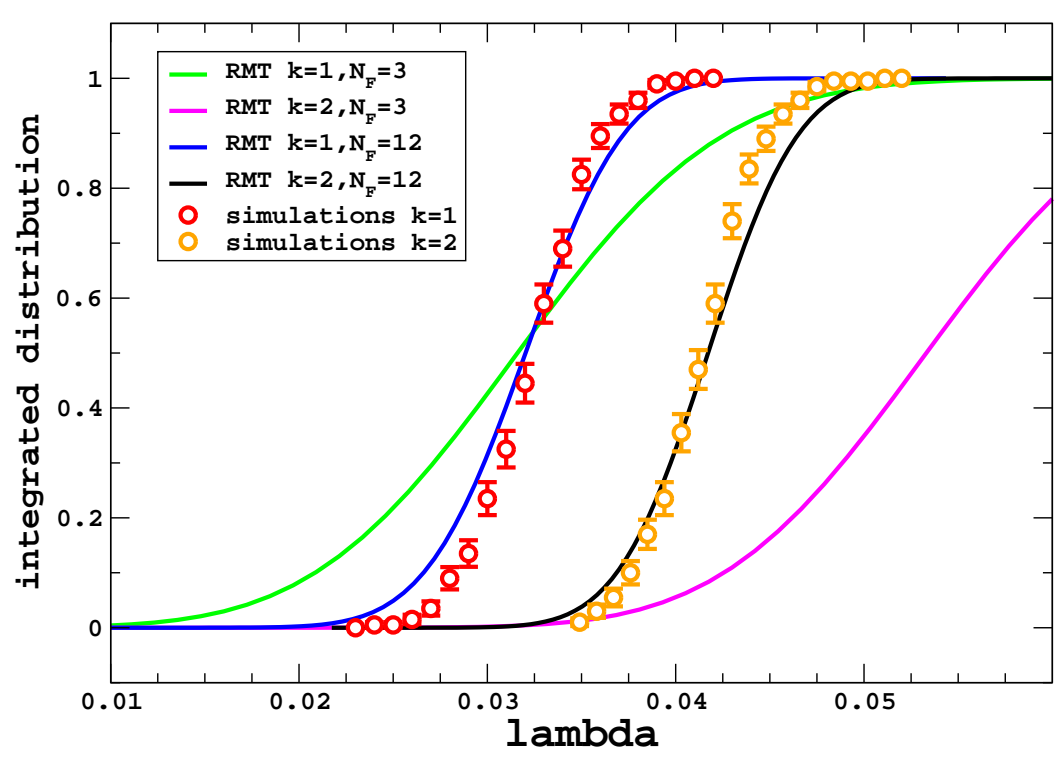

Figure 3: The integrated distribution of the two lowest eigenvalue quartets, from simulations of $n_{s}=3$ Asqtad staggered flavors. This is compared to RMT with $N_{f}=3$ and 12, corresponding to the strong and weak coupling limits.

\section{Simulations and analysis}

For $S U(3)$ gauge theory with quarks in the fundamental representation, various methods suggest that the critical number of flavors separating conformal and QCD-like behavior is between 8 and 12. In order to study this interesting region, we simulate $n_{s}=2$ and 3 staggered fermion flavors, corresponding to $N_{f}=8$ and 12 flavors in the continuum limit. (We do not take roots of the determinant of the staggered Dirac operator). We have also simulated $S U(3)$ gauge theory with $N_{f}=2$ flavors in the 2-index symmetric representation, using dynamical overlap fermions, which is described in [13]. We use the Asqtad staggered action [14], which includes improvements to reduce the violations of flavor symmetry ("taste breaking") at finite lattice spacing. This action is very well tested and has been heavily used in large scale simulations of lattice QCD [15]. There have been detailed comparisons of staggered eigenvalues with the Asqtad action to RMT [16], but only in the quenched approximation.

Because $n_{s}=2$ and 3 staggered flavors have not been simulated with this action before, a large scan of the parameter space of the bare couplings was required. Hence our first runs were on small volumes $10^{4}$, where we also gained experience on the dependence of the Hybrid Monte Carlo algorithm [17] on the quark mass and the discretization of the trajectory length. Once we generated large thermalized ensembles, we calculated the lowest eigenvalues of the Dirac operator using the PRIMME package [18]. In the continuum limit, the staggered eigenvalues form degenerate quartets, with restored flavor symmetry. In Figs. 2 and 3, we show the integrated distributions of the two lowest eigenvalue quartet averages,

$$
\int_{0}^{\lambda} p_{k}\left(\lambda^{\prime}\right) d \lambda^{\prime}, \quad k=1,2
$$




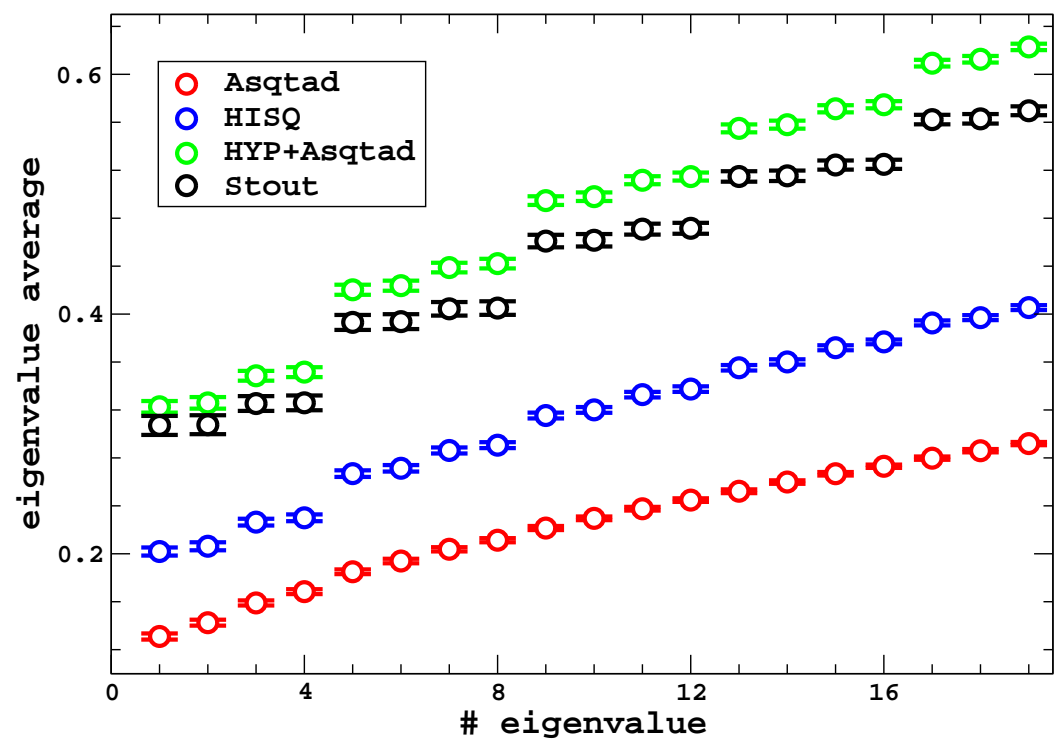

Figure 4: Comparison of different improvements of the staggered Dirac operator. The eigenvalues are calculated on the same ensemble of gauge configurations, which were generated using the Asqtad action.

for ensembles with $n_{s}=2$ and 3 staggered flavors respectively. Both simulations have quark mass $m a=0.01$, and the respective bare couplings are $\beta=3.9$ and 1.9. All low eigenvalues have small chirality, with no indication of non-zero topology. We see that the quark mass is less than the average smallest eigenvalue, which is necessary to probe the behavior of the eigenvalue distributions in the chiral limit. To compare with RMT, we vary $\mu=m \Sigma V$ until we satisfy

$$
\frac{\left\langle\lambda_{1}\right\rangle_{\mathrm{sim}}}{m}=\frac{\langle z\rangle_{\mathrm{rmt}}}{\mu}
$$

where $\left\langle\lambda_{1}\right\rangle_{\text {sim }}$ is the lowest quartet average from simulations and the RMT average $\langle z\rangle_{\text {rmt }}$ depends implicitly on $\mu$ and $N_{f}$. With this optimal value of $\mu$, we can predict the distributions $p_{k}\left(\lambda^{\prime}\right)$ and compare to the simulations.

In both cases, we see quite good agreement between simulations and RMT with the corresponding number of flavors in the continuum limit i.e. $N_{f}=8$ and 12. This is somewhat surprising. From the eigenvalues themselves, one can directly see that flavor breaking is significant, since degenerate quartets are not yet formed. A previous eigenvalue study used unimproved staggered quarks in dynamical fermion simulations [19]. They found excellent agreement with RMT but only if $N_{f}$ had the same value as the number of staggered flavors $n_{s}$. We also find that, at strong coupling, RMT with the continuum value $N_{f}=4 n_{s}$ does not describe the data. On coarse lattices, the flavor breaking is very large and only one pion can be tuned to the $\varepsilon$-regime for each staggered flavor. One has to go to weak coupling and finer lattices, where flavor breaking decreases, to recover the correct number of light pions.

These results indicate that both the $N_{f}=8$ and 12 flavor theories with fundamental quarks have a non-zero quark condensate $\Sigma$ i.e. chiral symmetry is spontaneously broken. If this conclusion holds against further studies of flavor breaking effects, our $N_{f}=8$ result will lend considerable 


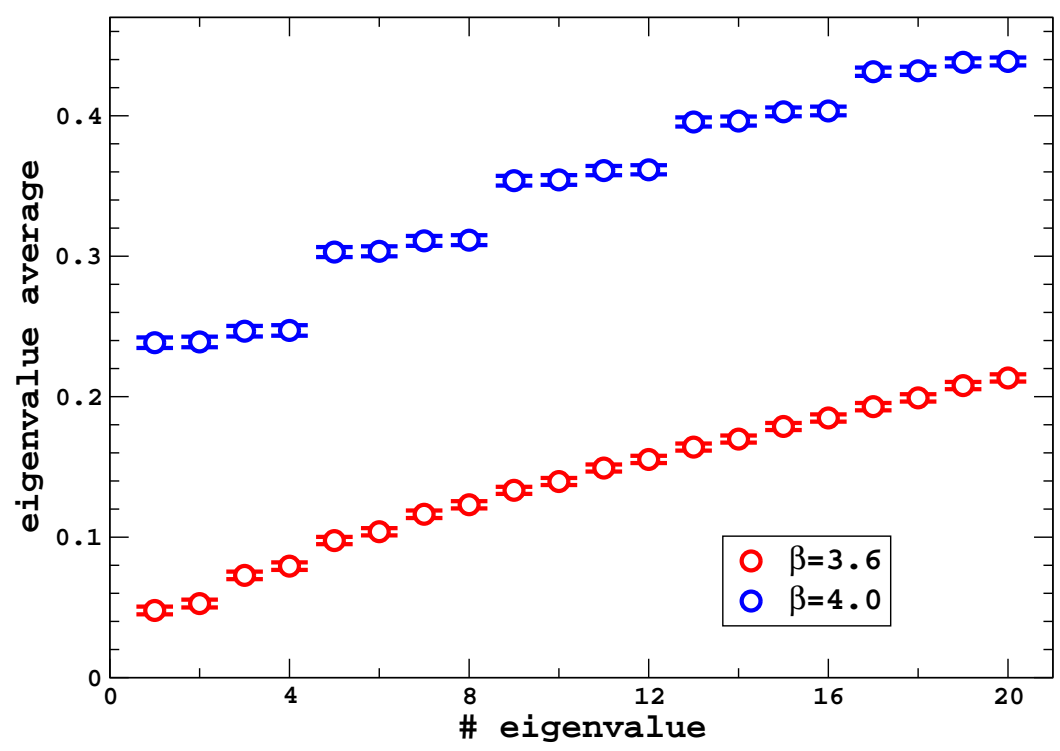

Figure 5: The lowest eigenvalues calculated on two ensembles with $n_{s}=1$ staggered flavor, with stout smearing used both in the sea and valence quark. The lattice volume is $12^{4}$.

support to the findings of [5, 元, but the $N_{f}=12$ spectrum would be inconsistent with the statement of [5] that this theory is conformal.

\section{Staggered improvement}

Since flavor breaking can have a dramatic effect on the eigenvalues, we are investigating various improvements of the staggered action, to bring the simulations closer to the continuum limit. In Fig. 月, we compare mixed actions, with gauge configurations generated using the Asqtad action, while the eigenvalues are those of various improved staggered Dirac operators. This figure is for $n_{s}=1$ staggered flavor at $\beta=6.8$ and volume $10^{4}$. The appearance of eigenvalue quartets which are clearly separated is a clear indication of reduced flavor breaking. Both HYP-smearing [20] and stout-smearing [21] seem to bring significant improvement relative to the Asqtad operator, while HISQ fermions [22] do not show as clear an improvement.

We also show in Fig. 5 the effect of using stout-smearing both in the sea and valence quark. As we go to weaker coupling towards the continuum limit, the eigenvalue quartet structure emerges clearly. Comparison of the improved eigenvalues with RMT is ongoing.

\section{Conclusions}

Knowledge of the conformal window is essential to build viable candidates of strongly interacting physics beyond the Standard Model, and lattice simulations will play a crucial role. Our technique of studying the eigenvalue properties complements other lattice approaches, such as calculating the beta function of the renormalized coupling, looking for finite-temperature transitions, or extracting the mass spectrum. This will hopefully lead to consensus about the nature of these 
new theories. Our first study gives an indication that $S U(3)$ gauge theory with $N_{f}=8$ and 12 flavors are both QCD-like, non-conformal theories. We are investigating various improvements to reduce flavor-breaking lattice artifacts and allow us to reach a stronger conclusion.

\section{Acknowledgments}

We thank Poul Damgaard for very helpful discussions, and Urs Heller who stressed the importance of reaching the quartet degeneracy limit. This research was supported by the DOE under grants DOE-FG03-97ER40546, DE-FG02-97ER25308, by the NSF under grant 0704171, by the DFG under grant FO 502/1 and by SFB-TR/55.

\section{References}

[1] S. Weinberg, Phys. Rev. D 19, 1277 (1979).

[2] L. Susskind, Phys. Rev. D 20, 2619 (1979).

[3] D. D. Dietrich and F. Sannino, Phys. Rev. D 75, 085018 (2007) [arXiv:hep-ph/0611341].

[4] S. Catterall and F. Sannino, Phys. Rev. D 76, 034504 (2007) [arXiv:0705.1664 [hep-lat]]; S. Catterall, J. Giedt, F. Sannino and J. Schneible, arXiv:0807.0792 [hep-lat].

[5] T. Appelquist, G. T. Fleming and E. T. Neil, Phys. Rev. Lett. 100, 171607 (2008) [arXiv:0712.0609 [hep-ph]].

[6] Y. Shamir, B. Svetitsky and T. DeGrand, Phys. Rev. D 78, 031502 (2008) [arXiv:0803.1707 [hep-lat]].

[7] A. Deuzeman, M. P. Lombardo and E. Pallante, arXiv:0804.2905 [hep-lat].

[8] L. Del Debbio, A. Patella and C. Pica, arXiv:0805.2058 [hep-lat].

[9] T. Banks and A. Casher, Nucl. Phys. B 169, 103 (1980).

[10] J. Gasser and H. Leutwyler, Phys. Lett. B 188, 477 (1987).

[11] E. V. Shuryak and J. J. M. Verbaarschot, Nucl. Phys. A 560, 306 (1993) [arXiv:hep-th/9212088].

[12] H. Fukaya et al., Phys. Rev. D 76, 054503 (2007) [arXiv:0705.3322 [hep-lat]].

[13] Z. Fodor, K. Holland, J. Kuti, D. Nogradi and C. Schroeder, PoS LAT2008, 058 (2008).

[14] K. Orginos, D. Toussaint and R. L. Sugar [MILC Collaboration], Phys. Rev. D 60, 054503 (1999) [arXiv:hep-lat/9903032].

[15] C. T. H. Davies et al. [HPQCD Collaboration and UKQCD Collaboration and MILC Collaboration and], Phys. Rev. Lett. 92, 022001 (2004) [arXiv:hep-lat/0304004].

[16] E. Follana, A. Hart, C. T. H. Davies and Q. Mason [HPQCD Collaboration and UKQCD Collaboration], Phys. Rev. D 72, 054501 (2005) [arXiv:hep-lat/0507011].

[17] S. A. Gottlieb, W. Liu, D. Toussaint, R. L. Renken and R. L. Sugar, Phys. Rev. D 35, 2531 (1987).

[18] A. Stathopoulos and J. R. McCombs, SIAM J. Sci. Comput., Vol. 29, No. 5, 2162 (2007).

[19] P. H. Damgaard, U. M. Heller, R. Niclasen and K. Rummukainen, Phys. Lett. B 495, 263 (2000) [arXiv:hep-lat/0007041].

[20] A. Hasenfratz and F. Knechtli, Phys. Rev. D 64, 034504 (2001) [arXiv:hep-lat/0103029].

[21] C. Morningstar and M. J. Peardon, Phys. Rev. D 69, 054501 (2004) [arXiv:hep-lat/0311018].

[22] E. Follana et al. [HPQCD Collaboration and UKQCD Collaboration], Phys. Rev. D 75, 054502 (2007) [arXiv:hep-lat/0610092]. 